\title{
Um modelo para o parecer psiquiátrico no hospital geral
}

\author{
A model for the written report in consultation-liaison psychiatry
}

\author{
Antonio Leandro Nascimento, Mônica Gomes de Andrade, Amanda Mendes Pinheiro \\ Assed e Marco Antonio Alves Brasil
}

\section{Resumo}

Objetivo: Propor um modelo estruturado para a elaboração das respostas às solicitações de pareceres psiquiátricos no hospital geral e discutir seus aspectos fundamentais. Métodos: Foi realizada uma pesquisa sistemática nas bases de dados PubMed/Medline e PsycINFO à procura de artigos que descrevessem modelos para a resposta aos pareceres psiquiátricos em hospitais gerais. Além disso, todos os artigos sobre psiquiatria de ligação publicados no Jornal Brasileiro de Psiquiatria (indexado no PsycINFO) nos últimos dez anos foram analisados para localizar as contribuições brasileiras ao tema. Foram selecionados artigos que descrevessem modelos estruturados para as respostas às solicitações de pareceres psiquiátricos em hospitais gerais. As informações contidas nesses artigos foram usadas na construção de uma proposta de ficha de parecer psiquiátrico. Resultados: Apenas dois artigos preenchiam os critérios de seleção. Outros cinco artigos que descreviam problemas no processo de consultoria psiquiátrica forneceram informações sobre questões que deveriam ser abordadas nos pareceres. Os modelos de parecer descritos foram analisados, e um novo modelo foi desenvolvido. Conclusão: Os pareceres psiquiátricos são documentos que atendem às necessidades médicas e educacionais em um hospital geral. Um modelo estruturado de parecer evita a omissão de informações importantes, melhorando a comunicação entre os psiquiatras pareceristas e os outros médicos no hospital geral.

Palavras-chave: psiquiatria de ligação, unidade hospitalar de psiquiatria, comunicação escrita.

\begin{abstract}
Objective: The aim of this article is to propose a structured model for the consultation-liaison psychiatry written report in general hospitals and discuss its fundamental aspects. Methods: The literature on this subject was reviewed through a systematic search at the PubMed/Medline and PsycINFO databases. Moreover, all the articles on consultation-liaison psychiatry published at Jornal Brasileiro de Psiquiatria (indexed at the PsycINFO) on the last ten years were analyzed to find the Brazilian contributions on this field. We selected the articles, which described structured models for the consultation-liaison psychiatry written report. Those articles were used as a basis for the development of a psychiatric expert report to be used in consultation-liaison settings. Results: We found two articles on consultation-liaison psychiatry written report models. Five other articles which described problems presented by consultationliaison psychiatry reports were used to gather data on issues that should be evaluated on the written reports. The consultation-liaison psychiatry written report models were analyzed and we developed a new written report model. Conclusion: Consultation-liaison psychiatry written reports are documents which fulfill medical and educational purposes at a general hospital. The development of a structured model for these reports might avoid lack of relevant data and improve doctor-to-doctor communication.
\end{abstract}

Key words: consultation-liaison psychiatry, general hospital psychiatric unit, written communication.

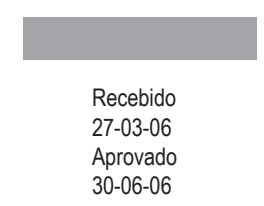

Instituto de Psiquiatria da Universidade Federal do Rio de Janeiro (IPUB/UFRJ) (Nascimento AL, Andrade MG, Assed, AMP)

Departamento de Psiquiatria da Faculdade de Medicina da UFRJ (Brasil MAA)

Correspondência para: Antonio Leandro Nascimento

Rua Aires Saldanha, 34/202 - Copacabana - 22060-030 - Rio de Janeiro-RJ - Tel.: (21) 2256-1087 - e-mail: antonioleandro@superig.com.br 


\section{Introdução}

A interconsulta psiquiátrica pode ser definida como uma subespecialidade da psiquiatria que se ocupa de assistência, ensino e pesquisa na interface entre psiquiatria e medicina (Lipowski, 1986). Em geral ela difere da prática psiquiátrica no tipo de abordagem ao paciente, no ambiente em que ocorre e pela intensa interação com outras especialidades (Querques e Stern, 2004). Existem dois modelos de interconsulta psiquiátrica: a psiquiatria de ligação, em que o psiquiatra faz parte de um serviço ou departamento como membro da equipe e/ou a consultoria psiquiátrica, em que o psiquiatra tem uma atuação episódica, identificando e tratando transtornos psiquiátricos.

Em um serviço de consultoria psiquiátrica, a comunicação entre médicos freqüentemente é feita através de pedidos de parecer com suas respostas. Logo, a eficiência desse serviço oscila de acordo com a comunicação escrita entre médicos, que é uma via de mão dupla (Williams e Wallace, 1974).

Dois estudos mostram a importância do parecer psiquiátrico no tratamento de pacientes atendidos por serviços médicos. O primeiro (Schnyder et al., 1997) mostrou que as informações obtidas através dos pareceres psiquiátricos de pacientes internados em hospitais gerais são freqüentemente transmitidas aos médicos responsáveis pelo atendimento ambulatorial aos pacientes. Esses dados estão presentes em até $84 \%$ dos boletins de alta, sendo a solicitação de um parecer (presente em $71,5 \%$ dos boletins de alta), o diagnóstico psiquiátrico $(66,7 \%)$ e o tratamento proposto $(76,2 \%)$ as informações mais freqüentemente relatadas. Outro estudo (Cotton e Ellis, 2001) mostrou que $85 \%$ dos clínicos gerais gostariam de receber os pareceres psiquiátricos sobre seus pacientes em um formulário estruturado no mesmo dia da avaliação, e que $83 \%$ dos clínicos gostariam que o psiquiatra fizesse a prescrição dos medicamentos psicotrópicos, caso fossem necessários.

Muitos estudos sobre o processo de consultoria como um todo já foram realizados, mas ainda há poucos artigos que abordem o documento do parecer psiquiátrico (Alexander $e$ Block, 2002). A liderança nessa área de pesquisa cabe aos ingleses (Bicalho et al., 1995) que, devido à organização de seu sistema de saúde em níveis hierárquicos baseados em referência e contra-referência, necessitam de um sistema eficiente de comunicação médica.

O parecer psiquiátrico é o registro da história, dos achados do exame e da impressão do psiquiatra acerca do paciente. São informações que devem ser reunidas de maneira clara e sucinta. Por outro lado, ele ainda inclui um plano terapêutico, embora não represente diretamente uma prescrição, e não trata apenas das preocupações do paciente, mas se refere principalmente às questões envolvendo o médico assistente $\mathrm{e}$ seu paciente (Garrick e Stotland, 1982). Sua função ultrapassa a condição assistencial, pois o parecer psiquiátrico também contribui para a educação e o treinamento dos profissionais que o solicitaram (Alexander e Block, 2002).

Durante a elaboração do parecer devemos levar em consideração que esse documento é formalmente dirigido ao médico assistente do paciente, mas também poderá ser lido por toda a equipe encarregada do paciente - que pode incluir enfermeiros, assistentes sociais, outros médicos e alunos -, por comissões de revisão de prontuários e pelo próprio paciente, visto que o prontuário lhe pertence (Garrick e Stotland, 1982).

Para elaborar o parecer, o psiquiatra deve conversar diretamente com o profissional que o solicitou, determinar o grau de urgência de sua resposta, rever o prontuário e os exames complementares, examinar o paciente, obter informações adicionais da família do paciente ou de outros informantes, estabelecer hipóteses diagnósticas e realizar os procedimentos necessários ao diagnóstico diferencial (solicitar exames complementares e obter mais informações sobre a história do paciente), assim como pode recomendar um tratamento (Kunkel et al., 2001).

Para Alexander e Bloch (2002), um parecer psiquiátrico deve responder a oito perguntas fundamentais sobre 0 paciente:

- quem é o paciente?

- por que foi solicitado um parecer psiquiátrico para esse paciente?

- 0 que encontramos ao examiná-lo?

- como é a família do paciente e seus outros suportes?

- o que nós concluímos a partir do exame?

- o que recomendamos para o tratamento?

- o que recomendamos no acompanhamento psicossocial?

- o que aconteceu depois do parecer?

O modelo de parecer que recomendamos responde a essas perguntas em suas sete seções (cabeçalho, motivo do pedido, anamnese, exame psíquico, exame físico, diagnóstico e recomendações) (Tabela 1).

\section{Métodos}

Foi realizada uma pesquisa bibliográfica nas bases de dados PubMed/Medline e PsycINFO a fim de localizar artigos que descrevessem modelos de respostas às solicitações de

Tabela 1. Perguntas fundamentais do parecer e seções em que serão abordadas

\begin{tabular}{ll}
\hline Perguntas fundamentais & Seções do parecer \\
\hline Quem é o paciente? & Anamnese (identificação) \\
Por que foi solicitado um parecer psiquiátrico para esse paciente? & Motivo do pedido \\
O que encontramos ao examiná-lo? & Exame psíquico, exame físico \\
Como é a família do paciente e seus outros suportes? & Anamnese (história pessoal) \\
O que nós concluímos a partir do exame? & Diagnóstico \\
O que recomendamos para o tratamento? & Recomendações \\
O que recomendamos no acompanhamento psicossocial? & Recomendações \\
O que aconteceu depois do parecer? & Evolução \\
\hline
\end{tabular}


pareceres psiquiátricos em hospitais gerais. Portanto foram realizadas pesquisas nas duas bases de dados, utilizando-se os termos general hospital psychiatry, consultation psychiatry, consultation-liaison psychiatry e psychiatric consultation combinados com written report. As bases de dados também foram pesquisadas através da combinação dos termos do Medical Subject Headings inerentes ao assunto. Não foram utilizados limites em relação às datas de publicação, ao tipo de artigo e à língua em que 0 artigo foi publicado. Além da pesquisa nas bases de dados, todos os artigos sobre psiquiatria de ligação publicados no Jornal Brasileiro de Psiquiatria (indexado no PsycINFO) nos últimos dez anos foram detalhadamente analisados à procura de contribuições brasileiras sobre o tema. Também se pesquisou a bibliografia citada pelos artigos selecionados, a fim de identificar novos artigos relevantes para o nosso estudo. Os modelos de respostas descritos foram analisados e então se desenvolveu um novo modelo de parecer.

\section{Resultados}

Foram encontrados dois artigos (Garrick e Stotland, 1982; Alexander e Block, 2002) que descreviam modelos de resposta aos pareceres psiquiátricos em hospitais gerais. Outros cinco artigos (Small e Fawzy, 1988; Carvalho et al., 1993; Bicalho et al., 1995; Souza, 1995; Cotton e Ellis, 2001) que discutiam problemas apresentados pelos pareceres em serviços de psiquiatria de ligação foram analisados para a construção de nosso modelo.

\section{Discussão}

Com base nos dados obtidos nos artigos selecionados, foi elaborado um modelo de parecer estruturado em sete seções (cabeçalho, motivo do pedido, anamnese, exame psíquico, exame físico, diagnóstico e recomendações) que será discutido pormenorizadamente a seguir. Foi elaborada uma ficha padronizada para resposta aos pedidos de parecer (Anexo 1). Nessa ficha, os campos em negrito são os campos a serem preenchidos e as instruções em itálico são as orientações sobre o preenchimento, que não devem ser reproduzidas na ficha para uso.

\section{Cabeçalho}

O cabeçalho deve permitir a fácil localização do parecer psiquiátrico entre as diversas anotações existentes no prontuário. Em seguida ao título, deve incluir uma informação sobre a evolução do processo (como primeira entrevista, avaliação inicial e evolução), a data e a hora de seu registro, bem como as fontes de informação consultadas naquela observação (entrevista com o paciente ou familiares, revisão de prontuário e resultados de exames complementares) (Garrick e Stotland, 1982).

\section{Motivo do pedido}

Os pareceres podem ser solicitados porque o médico assistente percebeu sintomas de transtornos psiquiátricos em seus pacientes, como humor deprimido e/ou comportamento agitado ou inadequado. Entretanto, a solicitação de um parecer pode indicar frustração, medo, culpa ou perturbação do médico assistente relacionados à sua dificuldade para diagnosticar, tratar, dar alta e/ou comunicar algo a um paciente (Garrick e Stotland, 1982).

Um psiquiatra de ligação experiente pode traçar hipóteses preliminares ao receber o pedido de parecer, conhecendo os diversos setores do hospital onde trabalha e suas particularidades, ou comparando o pedido que tem em mãos com os recebidos anteriormente, e então identificar os primeiros aspectos que deverá abordar. Entre os objetivos do psiquiatra, destacam-se o de remover os impedimentos e o de melhorar 0 entendimento e a relação entre paciente e médico assistente para que eles possam progredir com as medidas diagnósticas e terapêuticas.

Muitas vezes o motivo da solicitação do parecer não está claro no texto. Um estudo realizado em 1994 num ambulatório de interconsulta de um hospital geral (Bicalho et al., 1995) constatou que $65,8 \%$ dos pedidos de parecer não continham uma dúvida específica e que $35,36 \%$ foram recusados por serem ilegíveis ou não apresentarem informações fundamentais para o consultor, como a razão específica do pedido de parecer, os sintomas principais, a medicação e o tratamento aos quais o paciente está se submetendo, a opinião e o diagnóstico do solicitante. É fundamental falar diretamente com o profissional que solicitou o parecer para esclarecer suas dúvidas reais (Alexander e Bloch, 2002). Nessa seção deve-se indicar o profissional que solicitou o parecer, transcrever trechos do pedido de parecer, ou tentar traduzir esse pedido em uma frase que esclareça as demandas do serviço que solicitou o parecer, permitindo que o médico assistente possa explicar se seus desejos foram correspondidos com nossa avaliação ou se é necessário abordar outro aspecto do problema

\section{Anamnese}

A história do paciente a ser registrada no parecer é um relato, organizado cronologicamente, de todos os fatos relacionados diretamente ao problema do paciente (eventos médicos, sociais e interpessoais). Muitas vezes informações essenciais são omitidas no registro da história.

Em um estudo realizado em 1988, Small e Fawzy relataram que os seguintes dados são omitidos com mais freqüência: história familiar de doença psiquiátrica $(60,3 \%)$, história de abuso de substâncias $(44,9 \%)$, estado civil $(37,2 \%)$, uso prévio de psicotrópicos $(35,9 \%)$, tratamento psiquiátrico anterior $(26,9 \%)$ e história prévia de transtorno psiquiátrico $(24,4 \%)$.

Para que não haja omissões no registro da história, recomenda-se que ela seja divida em seções como identificação (nome do paciente, sexo, cor, naturalidade e procedência, data de nascimento, escolaridade, profissão, emprego atual, estado civil e religião); queixa principal e histórico da doença atual (incluindo a doença motivo do tratamento do paciente, e esclarecendo se essa é aguda ou crônica e qual o tratamento utilizado); histórias fisiológica, patológica pregressa, familiar e pessoal sem omitir os meios de suporte familiar e social do paciente.

O consultor deve considerar cuidadosamente quais informações podem ser incluídas no parecer e quais devem permanecer em sigilo. Essa discussão não exime o psiquiatra 
de registrar informações relevantes, como comportamento anti-social e transtornos psiquiátricos (Garrick e Stotland, 1982).

O uso da terminologia psiquiátrica é um ponto delicado do trabalho do consultor, que deve encontrar um meio-termo entre a simplificação excessiva e a ofuscação técnica. Em geral, como em toda redação, o objetivo é ser tão claro, direto e conciso quanto possível. Os termos técnicos devem ser empregados apenas quando necessários, e uma definição sucinta dos mesmos deve ser incluída no texto. O profissional solicitante do parecer está procurando a opinião de um especialista que ele possa entender e aplicar.

Outra característica do parecer psiquiátrico é a sua pretensão de esclarecer outros profissionais e estudantes sobre os aspectos psicossomáticos das doenças e estimular o entendimento de um paciente em sua singularidade. Uma avaliação psiquiátrica formal ajuda a atender a esses objetivos, sendo importante registrar tanto achados positivos quanto negativos. Contudo uma avaliação abrangente melhora a relação médico/paciente e será a base para a elaboração do diagnóstico e para as recomendações pertinentes.

\section{Exame psíquico}

O exame psíquico freqüentemente é ignorado. Seu registro pelo psiquiatra marca sua importância como elemento do trabalho médico e demonstra que os psiquiatras, como outros médicos, baseiam suas conclusões em uma série ordenada de avaliações.

Os seguintes aspectos devem ser avaliados: aparência, atitude, consciência, orientação, atenção, memória, inteligência, sensopercepção, fala, pensamento, humor, afeto, consciência do eu, vontade, pragmatismo, consciência de morbidade e planos para o futuro. Isso ajuda o consultor a se recordar e orienta a pessoa que pediu o parecer como organizar seu pensamento sobre o estado mental do paciente. É importante informar ao profissional que solicitou o parecer a influência do estado mental do paciente sobre o seu quadro clínico.

\section{Exame físico}

O exame físico é um aspecto freqüentemente negligenciado na elaboração dos pareceres psiquiátricos. Entretanto, ele pode ser de grande importância no diagnóstico diferencial dos transtornos mentais orgânicos e das manifestações psiquiátricas de doenças clínicas. 0 exame neurológico, por exemplo, freqüentemente é omitido ou realizado de maneira incompleta (Alexander e Block, 2002), porém a avaliação de um serviço de consultoria psiquiátrica em um hospital geral mostrou que ele foi necessário na avaliação de $24,52 \%$ dos pacientes (Souza, 1995). Além de ser fundamental à elaboração de alguns diagnósticos, o exame físico é uma maneira de aliviar a ansiedade que o paciente sente por ser examinado por um psiquiatra (Querques e Stern, 2004).

\section{Diagnóstico}

O problema do diagnóstico em psiquiatria é central (Lopes, 1983). Diagnósticos fazem parte da tradição médica, ajudam a estruturar um conjunto de observações para o diagnóstico diferencial e o tratamento. Entretanto, "os consultores psiquiátricos são freqüentemente vistos como intrusos, com conceitos vagos e estranhos" (Lipowski, 1986). Tal proposição pode ser confirmada por um estudo (Fortes, 1990) mostrando que psiquiatrias de um mesmo serviço de interconsultas utilizavam diagnósticos da nona versão da Classificação Internacional de Doenças (CID-9), do Manual Diagnóstico e Estatístico de Transtornos Mentais III (DSM-III), diagnósticos sindrômicos e relatos de queixas (nervoso) ou inespecíficos (poliqueixoso).

A padronização dos diagnósticos visa melhorar o atendimento aos pacientes, com o reconhecimento e o tratamento dos problemas mentais de forma mais adequada, permitir estudos epidemiológicos e pesquisas em interconsulta psiquiátrica (Carvalho et al., 1993).

A utilização de um sistema padronizado de diagnósticos como a CID-10 (OMS, 1996) é uma oportunidade para familiarizar os outros médicos com esse sistema de classificação, assim como é uma demonstração dos esforços da psiquiatria para formular diagnósticos mais precisos. Se não for possível fazer um diagnóstico, essa impossibilidade deve ser claramente explicada.

\section{Recomendações}

Essa seção é a única algumas vezes lida. Há dois tipos de recomendações: aquelas necessárias ao esclarecimento do diagnóstico (exames complementares; obtenção de detalhes da história, se do prontuário ou de um informante) e as prescrições, que podem incluir medidas farmacológicas, psicoterapêuticas, sociais, situacionais e legais. Pode ser necessário que o psiquiatra faça novas avaliações do paciente para verificar a resposta aos tratamentos prescritos e sua evolução. Essas visitas também devem ser registradas, seguindo o método descrito e anotando-se a evolução, as alterações no exame psíquico, no exame físico e nas recomendações.

0 parecer deve ser assinado e a assinatura deve explicitar a função da pessoa que respondeu ao parecer (se é interno, residente ou supervisor).

\section{Conclusão}

Um parecer psiquiátrico no hospital geral é uma ferramenta de grande utilidade no diagnóstico e no tratamento dos pacientes. Além disso, pode ser uma maneira de familiarizar médicos de outras especialidades com as características do diagnóstico e dos tratamentos psiquiátricos. Entretanto, para que o parecer cumpra essas funções, é necessário que seja escrito de maneira clara e precisa. A divisão do parecer em seções e o estabelecimento de itens a serem respondidos em cada uma facilitam a elaboração desse documento e evita falhas e omissões, tornando-o um instrumento mais eficiente. Mais estudos serão necessários para avaliar a utilidade desse modelo e sua adaptação a contextos específicos.

\section{Agradecimentos}

Os autores gostariam de agradecer aos doutores Mariana Pires da Luz e Cássio Alessandro Paganoti Sartório por seu inestimável auxílio na pesquisa bibliográfica. 


\section{Referências}

Alexander T, Block S. The written report in consultation-liaison psychiatry: a proposed schema. Aust N Z J Psychiatry, 36(2): 251-8, 2002.

Bicalho MR, Camargo IB, Contel JOB. Avaliação da comunicação médica: um estudo através dos pedidos de consulta à psiquiatria. J Bras Psiq, 44(12): 655-60, 1995.

Carvalho TFR, Lima MG, Ponde MP, Caetano D. Diagnóstico em interconsulta psiquiátrica: experiência de uma pesquisa realizada no HC-UNICAMP. J Bras Psiq 42(3): 157-60, 1993.

Cotton MA, Ellis D. How should a mental health liaison team communicate with general practitioners? Psychiatric Bulletin, 25(7): 278, 2001.

Fortes SL. Interconsulta psiquiátrica e ensino médico. In: Miguel Filho EC, Ramadam ZBA, Malbergier A, Souza DG. Interconsulta psiquiátrica no Brasil. Astúrias, p. 69-86, São Paulo, 1990.

Garrick TR, Stotland NL. How to write a psychiatric consultation. Am J Psychiatry, 139(7): 849-55, 1982.

Kunkel EJS, Monti DA, Thompson TL. Consultation, liaison and administration of a consultation-liaison psychiatry service. In: Wise MG, Rundell JR. Textbook of consultation-liaison psychiatry: psychiatry in the medically ill. 2 ed. Am Psychiatric Publising, p. 1324, 2001, Washington D.C.
Lipowski ZJ. Consultation-liaison psychiatry: the first half-century. Gen Hosp Psychiatry, 11: 1-22, 1986.

Lopes JL. Diagnóstico em psiquiatria. São Paulo: Editora Cultura Médica; 1983.

Organização Mundial da Saúde (OMS). Classificação Estatística Internacional de Doenças e Problemas Relacionados à Saúde: CID10. 3 ed. São Paulo: Edusp; 1996.

Querques J, Stern TA. Approach to consultation psychiatry: assessment strategies. In: Stern TA, Fricchione GL, Cassem NH, Jellinek MS, Rosenbaum JF, editors. Massachusetts General Hospital Handbook of General Hospital Psychiatry. Mosby, p. 9-19, Philadelphia, 2004.

Schnyder U, Feld C, Leuthold A, Buddenberg C. Reference to psychiatric consultation in the discharge letter of general hospital inpatients. Int J Psychiatry Med, 27(4): 391-402, 1997.

Small GW, Fawzy FI. Data omitted from psychiatric consultation notes. J Clin Psychiatry, 49(8): 307-9, 1988.

Souza JCRP. Consultoria psiquiátrica na Santa Casa de Campo Grande. J Bras Psiq, 4(3): 123-8, 1995.

Williams P, Wallace BB. General practitioners and psychiatrists: do they communicate? Br Med J, 1: 505-7, 1974. 
Parecer do Serviço de Psiquiatria

Visita $n^{0}$ Data:

Horário: Fontes:

Parecer solicitado por: indicar o nome do solicitante e a qual serviço está filiado.

Motivo do pedido: transcrever o motivo ou traduzi-lo em uma frase.

\section{Anamnese}

Identificação: nome do paciente, sexo, cor, naturalidade e local de procedência, data de nascimento, escolaridade, profissão, emprego atual, estado civil e religião.

Queixa principal: transcrever a queixa principal do paciente.

História da doença atual: relacionar em ordem cronológica os eventos médicos, sociais e interpessoais que influenciam o problema atual, incluindo os tratamentos prescritos.

História fisiológica: listar eventos como condições de gestação e parto, marcos do desenvolvimento neuropsicomotor (idade em que engatinhou, andou, falou, etc.), menarca, idade da primeira relação sexual e gestações.

História patológica pregressa: relatar as doenças apresentadas pelo paciente, os tratamentos empregados e a evolução. Não esquecer de listar a presença de transtornos psiquiátricos prévios (incluindo o tratamento) e história de abuso de substâncias.

História familiar: enumerar as doenças apresentadas pelos pais, irmãos e filhos dos pacientes. Não esquecer de listar transtornos psiquiátricos e seus tratamentos.

História pessoal: listar os eventos pertinentes da história da pessoa, relacionamentos, casamentos, condições de vida, hábitos e meios de suporte.

Paciente: Prontuário: Leito:

\section{Diagnóstico}

Registrar o diagnóstico do paciente baseado em um dos sistemas classificatórios vigentes (como a CID-1).

\section{Recomendações}

Listar as medidas necessárias ao diagnóstico (se houver), como realização de novos exames, obtenção de novos dados, etc. Prescrições: medidas farmacológicas, psicoterapêuticas, sociais e legais.

Parecer elaborado por: carimbo, indicação da função do parecerista (residente, supervisor, etc.) e assinatura.

\section{Exame psíquico}

Exame psíquico: registrar o exame psíquico do paciente.

Súmula psicopatológica: descrever os achados do exame psíquico nos seguintes aspectos: aparência, atitude, consciência, atenção, orientação, memória, inteligência, sensopercepção, fala, pensamento, humor, afeto, consciência do eu, vontade, pragmatismo, consciência de morbidade e planos para o futuro.

\section{Exame físico}

Exame físico: registrar o exame físico do paciente e não esquecer o exame neurológico. 